\title{
Optimization of the pulse width and injection time in a double-pass laser amplifier
}

\author{
Daewoong Park ${ }^{1}$, Jihoon Jeong ${ }^{1}$, and Tae Jun $\mathrm{Yu}^{1,2}$ \\ ${ }^{1}$ Department of Advanced Green Energy and Environment, Handong Global University, Pohang 37554, Korea \\ ${ }^{2}$ Global Institute of Laser Technology, Global Green Research and Development Center, Handong Global University, Pohang 37554, \\ Korea
}

(Received 13 July 2018; revised 4 October 2018; accepted 17 October 2018)

\begin{abstract}
We have optimized the input pulse width and injection time to achieve the highest possible output pulse energy in a double-pass laser amplifier using two Nd:YAG rods. For this purpose, we have extended the Frantz-Nodvik equation by simultaneously including both spontaneous emission and pump energy variation. The effective pump energy of the flash lamp was $8.84 \mathrm{~J}$ for each gain medium. The energy of $1 \mathrm{~J}$ could be amplified to an output energy of $12.17 \mathrm{~J}$ with the maximum achieved extraction efficiency of $63.18 \%$ when an input pulse having a pulse width of $168 \mu \mathrm{s}$ is sent $10 \mu \mathrm{s}$ after the absorbed pump energy becomes the maximum value.
\end{abstract}

Keywords: Frantz-Nodvik equation; input pulse optimization; pulse overlap; pump energy variation; spontaneous emission

\section{Introduction}

Among the various laser parameters, laser pulse width is a crucial factor to be selected carefully, depending on the application ${ }^{[1,2]}$. In particular, nanosecond and millisecond pulses have contributed significantly to applications in daily lives and practical laser work. For example, nanosecond lasers can be used for ultraviolet marking, thin film patterning and PC board cutting, as their pulse widths are in the moderate regime, offering a balanced throughput and high-quality material processing ${ }^{[2]}$. Microsecond laser pulses have been widely applied to various medical treatments ${ }^{[3-6]}$. Nd:YAG laser pulses with a width of microseconds and $1064 \mathrm{~nm}$ wavelength are useful for treating keloid, hypertrophic or surgical scars. Moreover, millisecond laser pulses have been used extensively in laser machining, e.g., for cutting, welding and drilling of machinery materials throughout the fields of automobile, shipbuilding and aerospace industries ${ }^{[7-9]}$. Recently, the high-energy laser system with pulse shaping techniques from nanosecond to millisecond timescales has been actively studied for these applications ${ }^{[10]}$. Therefore, as a tool for simulating laser amplifiers, the Frantz-Nodvik (F-N) equation needs to be extended to include diverse pulses without the limitation of their lengths and achieve more

Correspondence to: T. J. Yu, Department of Advanced Green Energy and Environment, Handong Global University, Pohang 37554, Korea. Email: taejunyu@handong.edu accurate results. In addition, as the user demands for laser applications grow, designing a laser amplifier that can maximize the output energy while minimizing the related costs is a vital issue. For achieving the latter, we employed a double-pass structure as a laser amplifier. This choice was made based on the following. First, in a double-pass amplifier - since the input pulse passes through a single gain medium twice - one gain medium has the same effect as using two. Therefore, it can aid the maximization of the input pulse energy. Second, because of requiring only half the amount of parts compared with amplifiers producing similar output energies, it can achieve a higher level of compactness and cost-effectiveness. Regarding the former, if the input pulse width reaches levels comparable to the fluorescence lifetime of the laser-active ions in the gain medium, spontaneous emission and pump energy variation must be considered during the amplification. However, there has been no research carried out considering spontaneous emission and pump energy variation simultaneously with the pulse overlap in a simulation of the pulse amplification. The $\mathrm{F}-\mathrm{N}$ equation has been used to calculate the amplified pulse energies for the arbitrary input pulses passing through the laser gain medium ${ }^{[11]}$. Since the spontaneous emission was neglected in the derivation of the equation, it can be applied only to calculations with this condition satisfied. Neglecting spontaneous emission during the amplification means that the population of the excited state can return to the ground state only through stimulated emission. 
For example, in the case of inputting an input pulse with a duration of $1 \mu \mathrm{s}$ into a Nd:YVO4 amplifier ${ }^{[12]}$, the fluorescence lifetime of the laser-active ions $(90 \mu \mathrm{s})$ is significantly longer than the duration of the amplification. Therefore, the pulse amplification ends before the excited ions spontaneously return to their ground state. In this case, the derivation conditions of the equation are satisfied. Besides, due to the short amplification time, no additionally incoming pump energy needs to be considered during the amplification. Strictly speaking, the existing F-N equation can only be applied if the input pulse width is significantly shorter than the fluorescence lifetime of the laser-active ions. In this paper, we have extended the $\mathrm{F}-\mathrm{N}$ equation to consider dynamically spontaneous emission and pump energy ignored during conventional simulations of the pulse amplification. The suggested method was applied to a flashlamp-pumped Nd:YAG double-pass amplifier structure, while simultaneously considering the pulse overlap effect as well. We changed the input pulse width and controlled its injection time. We have analyzed how these parameters can maximize the output pulse energy through our simulation results. Finally, we have verified that the optimal input pulse width and injection time can be realized within the given conditions to obtain the maximum output pulse energy.

\section{Modified Frantz-Nodvik equation}

Equation (1a) gives the modified $\mathrm{F}-\mathrm{N}$ equation suggested in our previous study, which enables the numerical calculation of the pulse amplification in a double-pass amplifier ${ }^{[13]}$. If the length of an input pulse exceeds the temporal length of the amplifier, as shown in Figure 1(a), the pulse overlap influences the amplification. Due to this pulse overlap, two inputs and two outputs appear to coexist in the gain medium. The overlapping degree between each input pulse is determined by $\tau_{1}, \tau_{2}$, which are calculated through the length of the gain media $L$, the distance between the gain media $d_{1}$, and the distance between the second gain medium and the mirror $d_{2}$. It can be represented by the time difference between the two input pulses entering the gain medium as shown in Figure 1(b). Taking the size of a typical amplifier into consideration, $\tau_{1}$ and $\tau_{2}$ are nanoseconds. Therefore, when the input pulse is a short pulse at the nanosecond level, the degree of overlap between the pulses is greatly influenced by $\tau_{1}$ and $\tau_{2}$. Depending on the values of $d_{1}$, $d_{2}$ and $L$, the pulses are partially or mostly overlapped in the gain medium. Also, as $d_{1}, d_{2}$ and $L$ become smaller, i.e., as $\tau_{1}$ and $\tau_{2}$ become smaller, the overlap between pulses becomes larger, resulting in a decrease of extraction efficiency and pulse-shape distortion. However, in the case of the microsecond or millisecond pulses, the pulse duration is remarkably longer than $\tau_{1}$ and $\tau_{2}$. Most of the input pulses are overlapped. Thus, the effects of $\tau_{1}$ and $\tau_{2}$ are relatively small compared to the case of the partial (a)

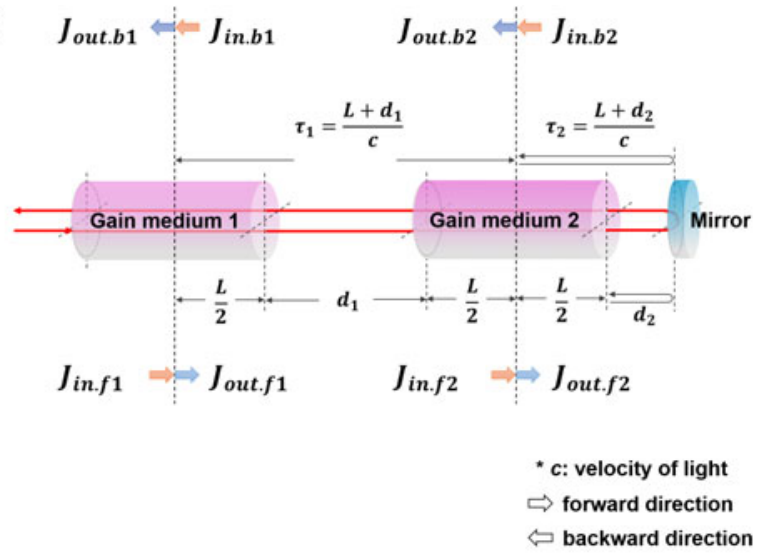

(b)

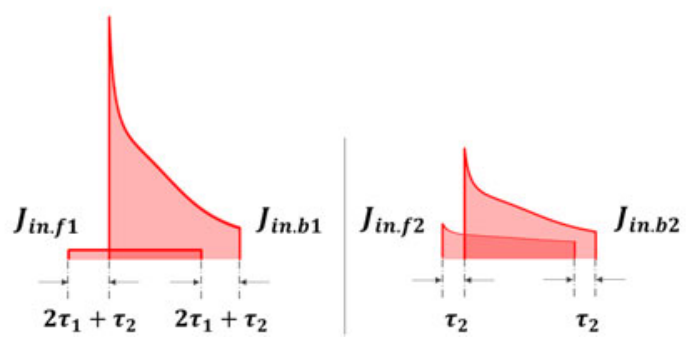

Figure 1. (a) Scheme of a double-pass laser amplifier: $J_{\text {in. } f 1}^{(n)}, J_{\text {out. } f 1}^{(n)}$, $J_{\text {in. } f 2}^{(n)}$ and $J_{\text {out. } f 2}^{(n)}$ are the equivalent fluences of the input and output energy in the forward propagating direction in gain media 1 and 2, respectively. Similarly, $J_{\text {in. } b 1}^{(n)}, J_{\text {out } b 1}^{(n)}, J_{\text {in. } b 2}^{(n)}$ and $J_{\text {out } b 2}^{(n)}$ are the equivalent fluences of the input and output energy in the backward propagating direction in gain media 1 and 2 , respectively. The temporal lengths $\tau_{1}$ and $\tau_{2}$ calculated from the geometry of the amplifier determine the time delay between each input. (b) The front part of the amplified input pulse is reflected on the mirror and is overlapped with the rear part of the input pulse in gain media 1 and 2, according to $\tau_{1}$ and $\tau_{2}$.

overlap. But there is also a decrease in extraction efficiency and pulse-shape distortion in comparison with single-pass amplification. Since there is a common gain medium for both inputs, it can be represented by the simple gain formula $\left(G_{E}=\left(J_{\text {out }} / J_{\text {in }}\right)\right)$, as in Equation (1a). Each output can be obtained by multiplying the respective input by the gain, as described in Equations (1b) and (1c):

$$
\begin{aligned}
G_{E}^{(n)} & =\frac{J_{\text {sat }}}{J_{\text {in. } f}^{(n)}+J_{\text {in. } b}^{(n)}} \\
\times \ln & \left\{1+\left[\exp \left(\frac{J_{\text {in. } f}^{(n)}+J_{\text {in. } b}^{(n)}}{J_{\text {sat }}}\right)-1\right] \exp \left(\frac{J_{\text {sto }}^{(n)}}{J_{\text {sat }}}\right)\right\} \\
J_{\text {out. } f}^{(n)}= & G_{E}^{(n)} J_{\text {in. } f}^{(n)}, \\
J_{\text {out. } b}^{(n)}= & G_{E}^{(n)} J_{\text {in. } b}^{(n)}, \\
J_{\text {sto }}^{(n)}= & J_{\text {sto }}^{(n-1)}-\left(G_{E}^{(n)}-1\right)\left(J_{\text {in. } f}^{(n-1)}+J_{\text {in. } b}^{(n-1)}\right) \\
J_{\text {sto }}^{(n)}= & J_{\text {sto }}^{(n-1)}-\left(G_{E}^{(n)}-1\right)\left(J_{\text {in. } f}^{(n-1)}+J_{\text {in } . b}^{(n-1)}\right) \\
& +I_{\text {pump }}^{(n-1)} \Delta T-\frac{1}{\tau_{f}} J_{\text {sto }}^{(n-1)} \Delta T .
\end{aligned}
$$


(a)

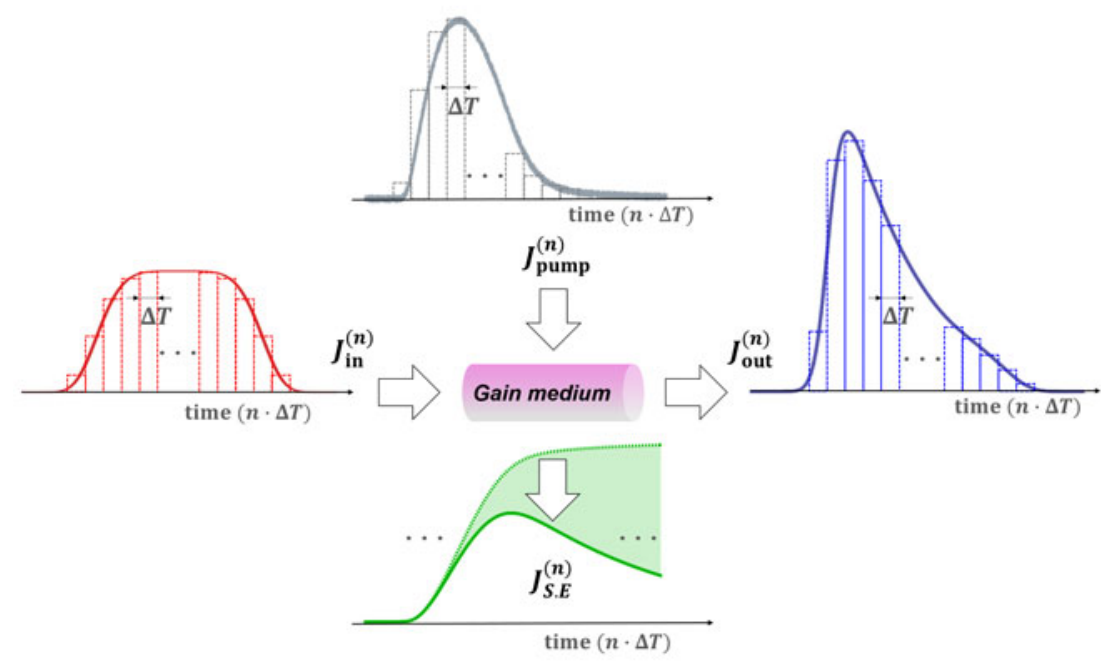

(b)

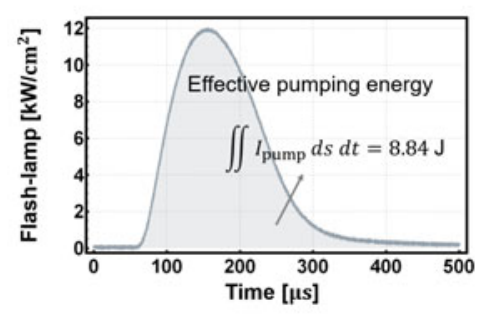

(c)

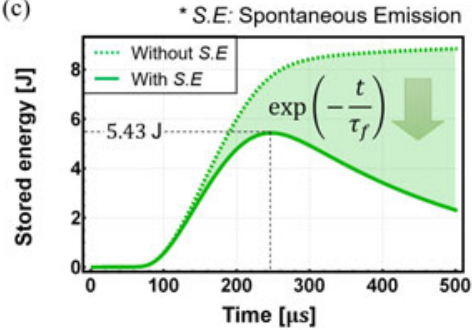

Figure 2. (a) Numerical F-N equation can be used to calculate the amplification during the whole duration of the input pulse according to the temporal sequence. $J_{\text {in }}^{(n)}, J_{\text {out }}^{(n)}, J_{\text {pump }}^{(n)}$ and $J_{S . E}^{(n)}$ are the input and output fluences, effective pump and spontaneous emission, respectively. (b) Temporal profile of the flash-lamp pulse whose effective pump energy is $8.84 \mathrm{~J}$. (c) Variation of the stored energy in the gain medium pumped by a flash lamp: the spontaneous emission shows an exponential attenuation described by the fluorescence lifetime $\left(\tau_{f}\right)$ of the laser-active ions. The solid green line was obtained by considering spontaneous emission, while the green dashed line without.

As shown in Figure 2(a), the input pulse is divided into rectangular-shaped pulses with discrete lengths of $\Delta T$. The superscript $n$ of each variable is the time index $(t=n$. $\Delta T, n=0,1,2, \ldots, N)$. The amplified fluence of the input pulse can be calculated in a time-sequential manner. Equation (1d) describes the conservation of the stored energy inside the gain medium. The equivalent fluence of the stored energy $J_{\text {sto }}^{(n)}$ in the laser gain medium represents the number density $\Delta_{0}$ of the excited laser-active ions described as small signal gain $\left(G_{0}=\exp \left(\Delta_{0} \sigma_{e} L\right)\right)$ in the original F-N equation. $\sigma_{e}$ and $L$ are the emission cross section and length of the gain medium, respectively. If the length of the input pulse is sufficiently short compared with the fluorescence lifetime of the laser-active ions, the equivalent fluence of the stored energy can be approximated to be influenced only by $J_{\text {in }}^{(n)}$ and $J_{\text {out }}^{(n)}$ representing the pulse amplification process as terms of stimulated emission. Thus, in Figure 2(a), the effective pump fluence $J_{\text {pump }}^{(n)}$ and the spontaneously emitted fluence $J_{S . E}^{(n)}$ can be neglected.

Meanwhile, in the case of pulsed Nd:YAG lasers with a pulse width of tens of microseconds, or even milliseconds, the input pulse is not sufficiently short compared with the fluorescence lifetime of the trivalent neodymium ions (230 $\mu \mathrm{s}$ ) used to dope the YAG crystal. The gray curve in Figure 2(b) gives the temporal shape of the flash-lamp pulse used in our lab (FL-58 type, EKSPLA, Lithuania), measured by an oscilloscope having $8.84 \mathrm{~J}$ of the effective pump energy. By assuming that spontaneous emission can be neglected, the laser gain medium would only absorb energy, without emitting the flash-lamp pump energy. Therefore, the pump energy would be accumulated along the green dashed line in Figure 2(c). However, as time passes, excited ions fall to an energy level above their ground state through spontaneous emission. Thus, the actual energy accumulated in the gain medium due to flash-lamp pumping and exponential decay described by the fluorescence lifetime follows the solid green line in Figure 2(c). Therefore, for the amplification of relatively long input pulses (in the microsecond to millisecond range) - which are comparable to, or longer than the fluorescence lifetime of $\mathrm{Nd}$ :YAG - in addition to the input and output, spontaneous emission and pump energy must also be considered dynamically during the calculation of the stored energy conservation. For the numerical approach, we assume that the values regarded as constants during a discrete time interval of $\Delta T$ affect the stored energy. Thus, the solution can be presented in a simple form, as given in Equation (1e).

In Equation (1e), the effective pump fluence $I_{\text {pump }}^{(n-1)} \Delta T$ can be obtained by scaling the pump pulse as the fluence unit $\left[\mathrm{J} / \mathrm{cm}^{2}\right]$ through the maximum stored energy in the gain medium and the time when it reaches its maximum value. If the input energy is sufficiently low, e.g., $J_{\text {in }} / J_{\text {sat }} \ll 1$ and moreover $G_{0}\left(J_{\text {in }} / J_{\text {sat }}\right) \ll 1$, small signal gain $\left(G_{0}=\right.$ $\left.\exp \left(J_{\text {sto }}^{(n)} / J_{\text {sat }}\right)\right)$ can be approximated by an overall amplifier gain $^{[14]}: G_{E}=J_{\text {out }} / J_{\text {in }}$. Thus, by measuring the amplifier gain for a low-energy input, the maximum equivalent fluence of the stored energy $J_{\text {sto.MAX }}^{(n)}$ can be obtained. In addition, the time resulting in maximum stored energy can be calculated by the convolution of the pump pulse and exponential decay given by the fluorescence lifetime of the laser-active ions. 
The spontaneous emission can be expressed as a partial time derivative of the number density of the excited state, $(\partial / \partial t) n(t)=-\left(1 / \tau_{f}\right) n(t)^{[14]}$. It means that the excited ions exhibit an exponential attenuation described by their fluorescence lifetime $\tau_{f}$. Therefore, the spontaneously emitted fluence can be described numerically by the last term of Equation (1e). The numerical amplification simulation technique of the laser pulse using the $\mathrm{F}-\mathrm{N}$ equation has been used extensively and it agrees well with experimental results $^{[15,16]}$. Also, the amplification simulation method of the laser pulse considering the overlap of pulses in the double-pass laser amplifier shows that the experimental results and the simulation results are well matched in the previous study ${ }^{[13,17]}$. Although spontaneous emission and the pump energy were newly considered in this study, these are added with the numerical terms based on the analytical formula. Thus, the proposed simulation method can be applied to the actual laser amplifier even without additional experiments.

\section{Simulation method and parameters}

The proposed method was applied to a double-pass amplifier with the aim of maximizing its output pulse energy. In Figure 1(a), we assumed its geometry as $L+d_{1}=1500 \mathrm{~mm}$, $L+d_{2}=1500 \mathrm{~mm}$. It was determined by considering the minimum time delay between the input pulses to include for the influence of the pulse overlap in the gain medium during the calculation. Two Nd:YAG rods having the following properties $-\emptyset=2.54 \mathrm{~cm}, \sigma_{e}=28 \times 10^{-20} \mathrm{~cm}^{2}, J_{\text {sat }}=$ $0.66 \mathrm{~J} / \mathrm{cm}^{2}$ at $1064 \mathrm{~nm}, \tau_{f}=230 \mu \mathrm{s}$ - were used as the laser gain media. The pump source was a flash lamp. We assumed that there was no dependence of flash-lamp pump energy absorbed in the laser rod on the longitudinal position of the laser rod and the two laser rods were pumped identically. Although the following is not included in this study, the pulse and gain media can be meshed into the spatial grids toward $z$, the direction of beam propagation, for more precise calculations ${ }^{[17]}$. Even with this method, it is necessary to assume that the energy distribution of each gain medium mesh is uniform. If the mesh size is sufficiently small, longitudinal gain distribution in the gain media can be calculated. Besides, the distribution of the amplified output beam and the residual pump beam in the transverse spatial domain can be calculated with two-dimensional F-N simulation ${ }^{[18,19]}$. We used super-Gaussian pulses of the same order, 5, for the amplifier input. Since the superGaussian input pulses have the same energy, $1 \mathrm{~J}$, when the pulse width increases, the peak intensity decreases, as shown in Figure 3. Thus, the input pulse width affects the shape and energy of the output pulse. The extraction efficiency $\left(\eta_{E}=\left(J_{\text {out }}-J_{\text {in }}\right) / J_{\text {pump }} \times 100 \%\right)$ and the variation of the stored energy in each $\mathrm{Nd}$ :YAG rod during the amplification

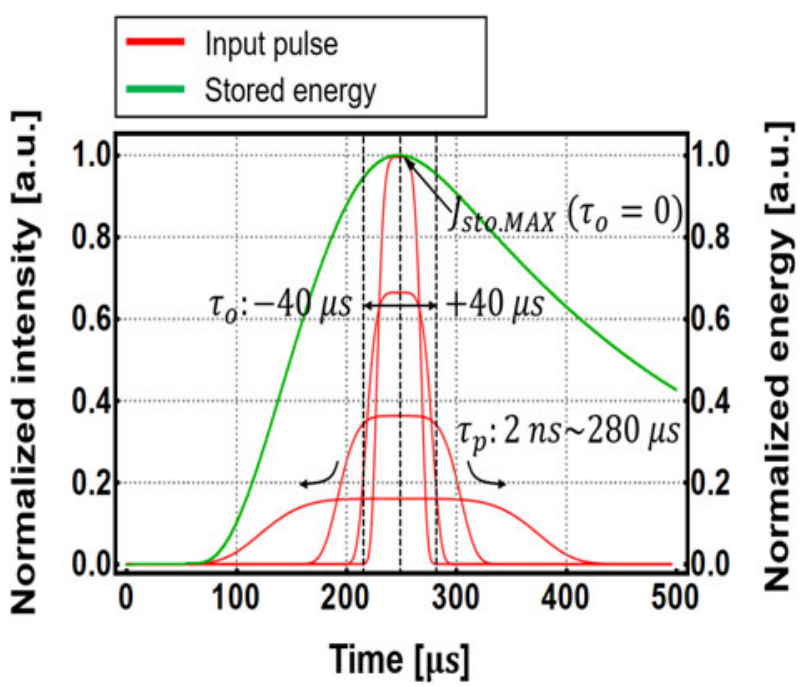

Figure 3. Simulation parameters: the input pulse width, given by its FWHM $\tau_{p}: 2 \mathrm{~ns}$ to $280 \mu \mathrm{s}$ (red) and the input pulse injection time, $\tau_{o}:-40 \mu$ s to $+40 \mu \mathrm{s}$ (dashed).

were scanned while varying the full width at half maximum (FWHM) $\tau_{p}$ and the injection time $\tau_{o}$ of the input pulse. The extraction efficiency indicates how much energy is originally extracted for amplification from the stored energy of the upper-laser level ${ }^{[14]}$. However, in the proposed calculation, the original extraction efficiency cannot be defined because spontaneous emission in addition to stimulated emission also affects the stored energy of the upper-laser level. Thus, the denominator of the extraction efficiency was replaced by the effective pump energy supplied by the flash lamp. We set the FWHM from $2 \mathrm{~ns}$ to $280 \mu \mathrm{s}$. The injection time was adjusted from $-40 \mu \mathrm{s}$ to $+40 \mu \mathrm{s}$ relative to $\tau_{o}$ set to zero: we set the injection time to zero when the stored energy in the Nd:YAG rod deposited by the flash lamp reaches its maximum, and it is positioned at the center of the input pulse as shown in Figure 3. We applied the maximum stored energy based on the measured value. The maximum stored energy in each entire laser rod was $5.43 \mathrm{~J}$ at $247 \mu \mathrm{s}$ after the flash-lamp emission as shown in Figures 2(b) and 2(c). A typically known xenon flash lamp converts approximately $50 \%$ of the electrical input power to radiated optical power. ${ }^{[14]}$ Therefore, half of the electrical energy $\left((1 / 2) C V^{2}\right)$ of $78.73 \mathrm{~J}$ from the power supply $(C: 60 \mu \mathrm{F}, V$ : $1620 \mathrm{~V}$, PS5053 series, EKSPLA, Lithuania) was converted into optical radiation of $39.37 \mathrm{~J}$ by the flash lamp (VQX$\mathrm{R}$ series, FLASHLAMPS, France). The maximum stored energy of $5.43 \mathrm{~J}$ is appropriate, considering that $30 \%-50 \%$ of the optical energy from the flash lamp is dissipated as heat, and some of the residual energy in the gain media is also lost due to spontaneous emission. Based on the measured value, the maximum efficiency of storing the electrical input energy in the Nd:YAG was about $6.9 \%$. 
(a)

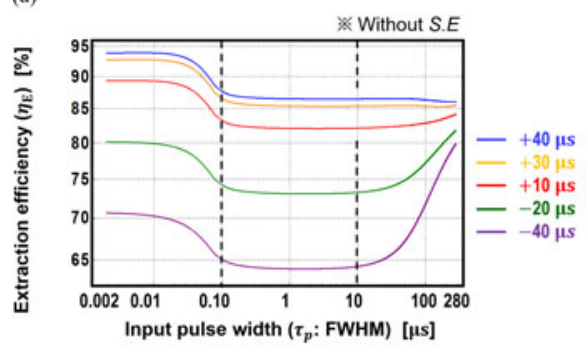

(d)

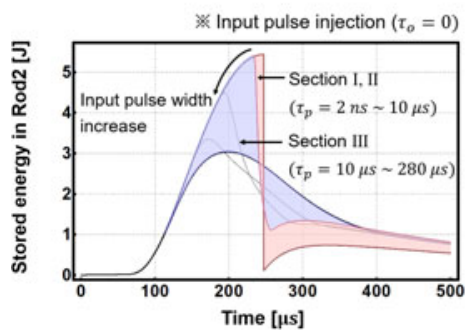

(b)

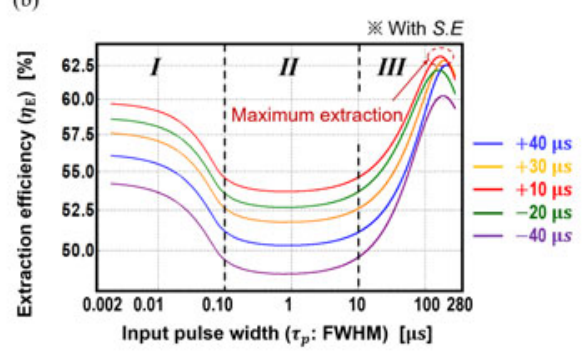

(e)

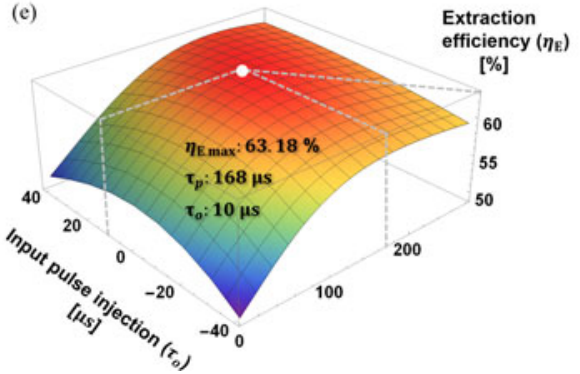

(c)

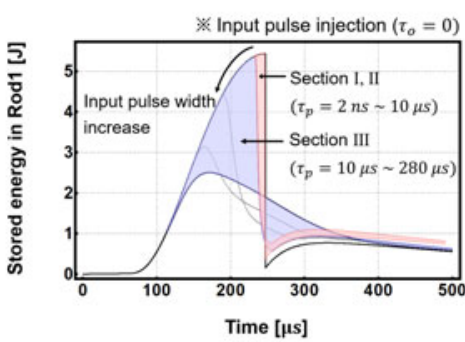

(f)

\% Input pulse energy / fluence $\left(1 \mathrm{~J} / 0.2 \mathrm{~J} / \mathrm{cm}^{2}\right)$ * Output pulse energy / fluence $\left(12.17 \mathrm{~J} / 2.4 \mathrm{~J} / \mathrm{cm}^{2}\right)$

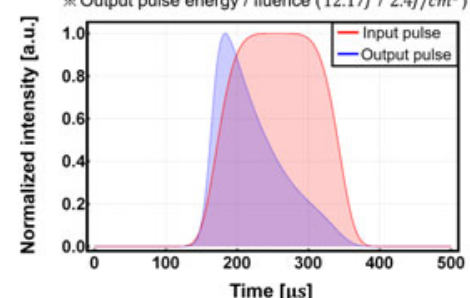

Figure 4. The extraction efficiency as a function of the input pulse width, where $\tau_{o}=+40 \mu \mathrm{s}(\mathrm{blue}), \tau_{o}=+30 \mu \mathrm{s}($ orange $), \tau_{o}=+10 \mu \mathrm{s}(\mathrm{red}), \tau_{o}=-20 \mu \mathrm{s}$ (green) and $\tau_{o}=-40 \mu \mathrm{s}$ (purple) (a) without considering spontaneous emission, while (b) with considering. (c), (d) Trend of the stored energy in Nd:YAG rod 1 and Nd:YAG rod2 during the amplification, where $\tau_{o}=0$ and $\tau_{p}=2$ ns-280 $\mu$ s. (e) Optimal pulse width and injection time of the input pulse resulting in the maximum extraction efficiency. (f) Normalized input and output pulse shapes at the optimal input conditions in (e).

\section{Simulation results and discussion}

Figure 4(b) shows the extraction efficiency $\eta_{E}$ variation as a function of the FWHM $\tau_{p}$ of the input pulse considering dynamically spontaneous emission and pump energy. Figure 4(a) does not consider spontaneous emission under the same conditions. The input pulse width was divided into three sections according to the trend of the extraction efficiency variation in Figure 4(b): Section I, $\tau_{p} \leqslant 100 \mathrm{~ns}$; Section II, $100 \mathrm{~ns} \leqslant \tau_{p} \leqslant 10 \mu \mathrm{s}$; Section III, $10 \mu \mathrm{s} \leqslant$ $\tau_{p} \leqslant 280 \mu \mathrm{s}$. Regarding every injection time $\tau_{o}$ of the input pulse, the extraction efficiency for the given input pulse width tends to show similar variations in all three sections, i.e., the maximum extraction efficiency occurs in the region where the pulse width is longer than $100 \mu \mathrm{s}$. Therefore, we have indicated only the $\tau_{o}$ values of $+40 \mu \mathrm{s}$ (blue), $+30 \mu \mathrm{s}$ (orange), $+10 \mu \mathrm{s}$ (red), $-20 \mu \mathrm{s}$ (green), and $-40 \mu \mathrm{s}$ (purple). From Figures $4(\mathrm{a})$ and $4(\mathrm{~b})$, it can be seen how significant the effect of spontaneous emission is in a laser pulse amplification. The most important thing is the calculation difference that the extraction efficiency is always higher in the case of not considering spontaneous emission because of the assumption that all the pump energy is absorbed by the gain medium without emitting. As the calculation in Figure 4(b), the overlap of pulses in the double-pass amplifier is also considered in Figure 4(a). Thus, at the level below $100 \mathrm{~ns}$, as the pulse width increases, the extraction efficiency decreases. But there is no loss due to spontaneous emission; the extraction efficiency increases again with longer input pulse width. Therefore, as the pulse width becomes similar to or longer than the fluorescence lifetime of the laser-active ions, the effect of spontaneous emission should be considered in the pulse amplification. From the results applying the proposed calculation method as described in Figure 4(b), dynamic consideration of spontaneous emission and pump energy during the amplification can yield the following noteworthy results.

In Sections I and II, the input pulse width is remarkably short compared with the fluorescence lifetime of Nd:YAG. Since the amplification time is concise, the stored energy variation of the gain medium is mainly influenced by the stimulated emission and the extraction efficiency depends on the pulse overlap effect in the double-pass amplifier rather than spontaneous emission and pump energy variation. In Section I, the shorter the pulse width, the higher the extraction efficiency because the pulse overlap in the doublepass amplifier is reduced, leading to an increase in the output pulse energy. On the other hand, the extraction efficiency tends to stay almost constant with a varying input pulse width in Section II. As the input pulse width is longer, larger energy is lost due to the pulse overlap, but the pump energy that could not be used in short pulse amplification can be utilized due to extended amplification time. Therefore, this has the effect of increasing the output pulse energy. That is why the extraction efficiency gradually decreases and remains constant as the input pulse width increases throughout Sections I and II. Consequently, when the input pulse width is within a sufficiently short range compared 
with the fluorescence lifetime of Nd:YAG, a pulse as short as possible is advantageous for high extraction efficiency with reducing overlap loss and rapid depletion of stored energy in gain media as shown in Figures 4(c) and 4(d). In addition, to increase the extraction efficiency, the input pulse should be injected when the energy stored in the gain medium through pumping is at its maximum. Thus, as shown in Figure 4(b), the closer the pulse injection is to $\tau_{o}=0$, the higher the extraction efficiency.

Differently from Sections I and II, in Section III, the input pulse width becomes comparable to the fluorescence lifetime of Nd:YAG. Hence, the pulse overlap effect, spontaneous emission, and pump energy variation are complexly involved in the extraction efficiency. On the left-hand side of Section III in Figure 4(b), the extraction efficiency tends to increase as the input pulse width gradually becomes larger. In this case, the effect of using more pump energy by longer amplification time has a more dominant influence on the output pulse energy than the energy loss caused by pulse overlap and spontaneous emission. On the contrary, the right-hand side of Section III in Figure 4(b) shows an opposite trend. Thus, the extraction efficiency reaches its maximum value and then decreases. As a result, in Section III, we could find the optimal injection time and input pulse width, with which the maximum extraction efficiency is achieved. The optimal injection time $\tau_{o}$ was $+10 \mu \mathrm{s}$ and the optimal FWHM $\tau_{p}$ was $168 \mu \mathrm{s}$ as marked in Figure 4(e). From the considered simulation parameters and conditions of the amplifier, the maximum extraction efficiency was $63.18 \%$. The maximum achievable output pulse energy and fluence through the input pulse energy of $1 \mathrm{~J}$ and effective pump energy of $8.84 \times 2 \mathrm{~J}$ were $12.17 \mathrm{~J}$ and $2.4 \mathrm{~J} / \mathrm{cm}^{2}$, respectively as shown in Figure 4(f).

\section{Conclusions}

We have studied the design of a laser amplifier that can maximize its output pulse energy. For this purpose, we have chosen a double-pass amplifier structure that can utilize one laser gain medium twice, leading to economic benefits of its manufacturing and a compact design. Second, a pulse amplification simulation method, considering spontaneous emission and pump energy variation numerically, has been proposed by extending the F-N equation. Finally, we set the simulation parameters to the super-Gaussian input pulse width and the injection time, while considering the pulse overlap effect during the amplification. We observed the extraction efficiency and the stored energy variation in the gain medium to obtain the highest achievable output pulse energy. With the considered parameters, for pulse widths below 100 ns, i.e., for pulse widths significantly shorter than the fluorescence lifetime of the laser-active ions, the result fulfilled our intuitive expectations. Shorter pulses injected when the stored energy of the gain medium becomes maximum can produce higher possible output pulse energy through abrupt depletion of the upper-state ions while reducing the energy wasted by spontaneous emission and temporal overlap. Furthermore, as an intermediate region, input pulse widths between $100 \mathrm{~ns}$ and $10 \mu \mathrm{s}$ do not exhibit a noteworthy influence on the output pulse energy. Finally, beyond $10 \mu \mathrm{s}$ pulse widths, we obtained the optimal input pulse width and injection time resulting in the maximum output pulse energy. Sometimes the original F-N equation is limited for the input pulse width range in specific amplifier conditions by the assumptions made for its derivation, e.g., neglecting spontaneous emission. The proposed method allows for a more accurate amplification simulation without the limitation of pulse widths by considering dynamically spontaneous emission and pump energy. Therefore, even if the structure and conditions of the amplifier are different from the demonstrated Nd:YAG double-pass structure, the method is applicable to other amplifiers and enables us to get another meaningful result. Consequently, this paper is expected to be useful for designing pulsed laser amplifiers with the aim of generating more energy under the same conditions.

\section{Acknowledgements}

This work was supported by the Industrial Strategic Technology development program, 10048964. The development of the $125 \mathrm{~J} \cdot \mathrm{Hz}$ laser system for laser peening was funded by the Ministry of Trade, Industry \& Energy (MI, Korea).

\section{References}

1. R. D. Schaeffer, Ind. Laser Solut. Manuf. 32, 1 (2017).

2. R. Patel, J. Bovatsek, and H. Chui, Ind. Laser Solut. Manuf. 32, 3 (2017).

3. A. Rossi, R. Lu, M. K. Frey, T. Kubota, L. A. Smith, and M. Perez, J. Drugs Dermatol. 12, 11 (2013).

4. A. E. S. A. E. H. Al-Mohamady, S. M. A. Ibrahim, and M. M. Muhammad, J. Cosmet. Laser Ther. 18, 4 (2016).

5. N. Ezra, A. Arshanapalli, R. Bednarek, S. Akaishi, and A. K. Somani, J. Cosmet. Laser Ther. 18, 4 (2016).

6. M. L. Siniaeva, M. N. Siniavsky, V. P. Pashinin, A. A. Mamedov, V. I. Konov, and V. V. Kononenko, Laser Phys. 19, 5 (2009).

7. A. Choubey, R. K. Jain, S. Ali, R. Singh, S. C. Vishwakarma, D. K. Agrawal, R. Arya, R. Kaul, B. N. Upadhyaya, and S. M. Oak, Opt. Laser Technol. 71, 6 (2015).

8. N. Kumar, M. Mukherjee, and A. Bandyopadhyay, Opt. Laser Technol. 88, 24 (2017).

9. H. Wang, H. Lin, C. Wang, L. Zheng, and X. Hu, J. Eur. Ceram. Soc. 37, 4 (2017).

10. R. Meijer, A. Stodolna, K. Eikema, and S. Witte, Opt. Lett. 42, 14 (2017).

11. L. M. Frantz and J. S. Nodvik, J. Appl. Phys. 34, 8 (1963).

12. M. J. Yarrow, J. W. Kim, and W. A. Clarkson, Opt. Commun. 270, 2 (2007). 
13. J. Jeong, S. Cho, and T. J. Yu, Opt. Express 25, 4 (2017).

14. W. Koechner, Solid-State Laser Engineering (Springer, New York, 2006).

15. J. Sung, S. Lee, T. Yu, T. Jeong, and J. Lee, Opt. Lett. 35, 18 (2010).

16. T. Yu, S. Lee, J. Sung, J. Yoon, T. Jeong, and J. Lee, Opt. Express 20, 10 (2012).
17. M. Li, X. M. Zhang, Z. G. Wang, X. D. Cui, X. W. Yan, X. Y. Jiang, J. G. Zheng, W. Wang, and M. Li, Opt. Commun. C 382, 49 (2017).

18. T. A. Planchon, F. Burgy, J.-P. Rousseau, and J.-P. Chambaret, Appl. Phys. B 80, 6 (2005).

19. S. Hwang, T. Kim, J. Lee, and T. Yu, Opt. Express 25, 9 (2017). 